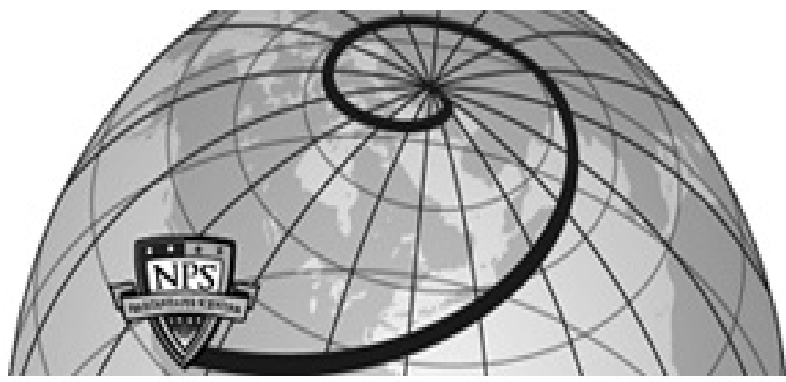

Calhoun: The NPS Institutional Archive DSpace Repository

\title{
Laser beam-quality/aperture-shape scaling relation
}

Rockower, Edward B.

https://hdl.handle.net/10945/44117

This publication is a work of the U.S. Government as defined in Title 17, United States Code, Section 101. Copyright protection is not available for this work in the United States.

Downloaded from NPS Archive: Calhoun



http://www.nps.edu/library
Calhoun is the Naval Postgraduate School's public access digital repository for research materials and institutional publications created by the NPS community. Calhoun is named for Professor of Mathematics Guy K. Calhoun, NPS's first appointed -- and published -- scholarly author.

Dudley Knox Library / Naval Postgraduate School 411 Dyer Road / 1 University Circle Monterey, California USA 93943 


\title{
Laser beam-quality/aperture-shape scaling relation
}

\author{
Edward B. Rockower
}

\begin{abstract}
Many high-energy lasers (HELs) have noncircular output apertures. Some are rectangular in shape with or without a central or noncentral (up to $30 \%$ ) obscuration. However, most high-energy laser propagation codes (especially those developed for systems analysis) model the aperture as either an unobscured circle or as a circle with fixed (e.g., 10\%) obscuration. We present a beam-quality/aperture-shape scaling relation which can be useful when applying these codes to realistic designs for HELs. Our analysis also yields a generalized formula for angular size of the Airy disk and definitions of a characteristic aperture length and aperture quality.
\end{abstract}

\section{INTRODUCTION}

Development of computer codes for nonlinear laser beam propagation through turbulent atmospheric conditions, including smoke and dust, has required (and received) a large amount of modeling effort (see e.g., Ref. 1). However, other important features of high-energy lasers (HELs) include the rectangular aperture and large (possibly up to $30 \%$ ) central obscuration. The latter is characteristic of unstable resonator designs common to most high-energy lasers.

Two major categories of computer code have been developed for modeling HEL propagation:

(1) Fundamental wave optics codes: these codes start from the basic microscopic laws of E-M radiation and implement various phenomenological models of the atmospheric turbulence and particulates (e.g., Ref. 2).

(2) Moderate accuracy system-level scaling law codes: these codes start from phenomenological and analytical approximations to the $\mathrm{E}-\mathrm{M}$ wave equations and attempt to represent the details of propagation through the atmosphere with a few parameters (e.g., a phase integral) based on integrated properties of the atmospheric conditions, light intensity, wave length, etc. These codes are based on experimentally and numerically (from the fundamental wave optics codes) derived data bases. ${ }^{3}$

One limitation is common to many laser propagation codes (especially the system's analysis codes), namely, the code may handle only circular laser apertures. This limitation has not stopped application of the codes outside their range of validity.

In attempting to exercise such a propagation code to simulate performance of rectangular aperture lasers, it is reasonable (because of possible nonlinear effects) to try to match the output power and beam intensity of

The author is with Naval Postgraduate School, Department of Operations Research, Monterey, California 93943.

Received 12 September 1985. the real laser. If the former condition is met, the latter is equivalent to requiring that the area of the circular aperture be the same as for the real laser, and, therefore,

$$
\pi D^{2} / 4=A,
$$

where $A$ is the rectangular aperture area (excluding obscured regions) and $D$ is the circular aperture diameter.

The following analysis estimates the adequacy of modeling realistic HEL apertures as circular apertures and leads to the derivation of a beam-quality/aperture-shape scaling relation. A generalization of the Airy disk size and shape-dependent characteristic aperture-length and aperture-quality parameters are presented based on this analysis. We then apply the scaling relation to one of the system's analysis propagation codes to demonstrate an application of the scaling relation.

\section{Derivation of the Scaling Relation}

An asymptotic approximation formula for estimating the fraction of encircled energy within a given radius in a plane perpendicular to the direction of propagation has been derived for imaging systems with oddly shaped apertures. ${ }^{4}$ Modulation transfer function (MTF) techniques were applied to a uniformly illuminated aperture having arbitrary shape and obscurations resulting in the following formula:

$$
E(r)=1-\lambda f R /\left(2 \pi^{2} r\right)
$$

where $\lambda=$ wavelength

$f=$ effective focal length,

$r$ = radial dimension in the focal plane,

$R=$ HEL aperture perimeter/area ratio, and

$E=$ normalized encircled energy, i.e., the fraction of the energy transmitted by the aperture that falls within a circle of radius $r$ about the geometrical focal point.

The main feature of this result for our purposes is that, apart from the laser wavelength and system focal distance, the fraction of encircled energy depends only on the ratio $R / r$. Hence it is independent of details of 
the aperture shape apart from the value of $R$. In the derivation it is assumed that the laser beam propagates in a linear medium with no effects of atmospheric fluctuations, wind, etc. We see from Eq. (2) that the radius $r$ encircling a given fraction of energy increases linearly with $R$.

A related effect results from imperfections of the laser wave front at the point where the beam leaves the laser aperture. A degraded beam quality (phase front distortion) is often represented with a beam quality parameter $M$. The value of $M$ is always greater than or equal to 1 and is given as times diffraction-limited; i.e., the beam spread is $M$ times the diffraction-limited rate of beam divergence. Most, if not all, propagation codes allow the user to set $M$. If $E(r)$ is the encircled energy function when $M$ is equal to one, for arbitrary $M$, the encircled energy becomes

$$
E^{\prime}(r)=E(r / M) \text {. }
$$

Combining the two results for beam spread, we obtain

$$
E^{\prime}(r)=1-(\lambda M R)(f / r) /\left(2 \pi^{2}\right) .
$$

From Eq. (4) we identify a method of partially compensating for rectangular apertures and various sizes and types of obscuration. We see that the asymptotic spread of light emanating from two laser apertures having the same value of the product $\lambda M R$ will be the same. Hence a better approximation to an arbitrary aperture results if we require

$$
M R=M^{\prime} R^{\prime} .
$$

Two lasers with the same value of $M^{*} R$, power, and aperture area (hence the same intensity) will have approximately the same asymptotic beam divergence. This is the main result of this section.

This aperture-shape/beam-quality similarity (or scaling) relation is to be distinguished from the socalled similarity law of diffraction, ${ }^{5}$ which compares diffraction from two apertures of geometrically similar shape but different size.

Having two parameters for the equivalent circular aperture at our disposal $\left(M^{\prime}\right.$ and $\left.D^{\prime}\right)$ allows us to match both area and ratio $R$ of any HEL aperture. For example, we can model a circular aperture with a central obscuration with another circular aperture of the same area and output power and with beam quality $M^{\prime}$ satisfying Eq. (5). These requirements lead to

$$
\begin{aligned}
M^{\prime} & =M(1+\sqrt{ } F) / \sqrt{ }(1-F), \\
D^{\prime} & =D \sqrt{ }(1-F),
\end{aligned}
$$

where $F$ is the fraction obscuration.

It is easy to derive similar equations for rectangular apertures with and without (rectangular) obscurations. The results for the latter and for circular apertures are presented in graphic form in Fig. 1. Each curve gives the beam quality of an equivalent uniform circular aperture with the same area as the rectangular aperture whose length to width $(L / W)$ ratio is shown to the right of the curve. The curve for a circular aperture with obscuration $F$ is also presented.
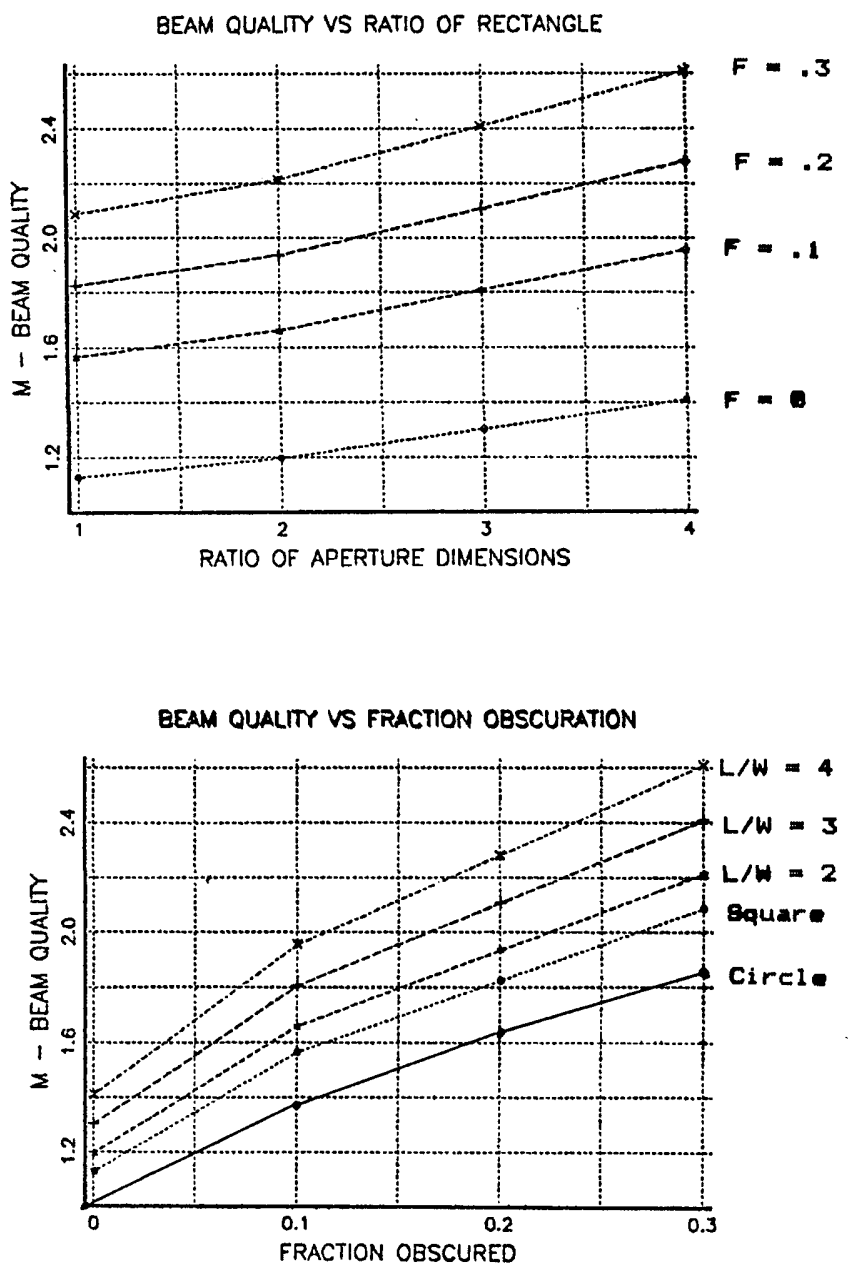

Fig. 1. Equivalent beam quality as a function of length/width ratio $(L / W)$ and fraction obscuration $F$.

Note that any aperture can be characterized by the ratio $R / R^{\prime}$. This is the value of the dimensionless parameter $M^{\prime}$ satisfying Eqs. (5) and (1) with $M$ set to one. Since the circle gives the minimum beam spread for a given aperture area (the minimum value of $R$ ), $M^{\prime}$ (i.e., $R / R^{\prime}$ ) indicates the (inverse of) beaming effectiveness of the aperture, i.e., the actual beam spread relative to this ideal (achieved for a circular aperture and perfect beam quality). We propose calling this quantity the aperture quality in close analogy with beam quality. For example, a square aperture has an aperture quality of $2 / \sqrt{ } \pi$. The aperture quality, like beam quality, is always greater than one. An alternative interpretation of Fig. 1 is as a plot of aperture quality (the ordinate) for various aperture shapes. With this definition of aperture quality, Eq. (5) can be stated in words as "two apertures having the same product of beam quality times aperture-quality will produce the same asymptotic encircled energy function."

\section{Characteristic Length Parameter and Diffractive Beam Spread}

For an unobscured circle the ratio of perimeter to area is 


$$
R=4 / D,
$$

and for a square with side $a$,

$$
R=4 / a \text {. }
$$

This suggests defining a parameter $L$, the characteristic linear dimension of an arbitrarily shaped aperture as

$$
L=4 / R \text {. }
$$

Reference to our scaling relation, Eq. (5), suggests that for arbitrary $M$ an effective aperture dimension is

$$
L^{\prime}=4 /(M R) \text {. }
$$

For a rectangle of sides $a$ and $b, R$ is found to be

$$
R=2(a+b) /(a b) .
$$

Hence for a rectangle the characteristic length (if $M=$ 1 ) is

$$
L=2 a b /(a+b)
$$

or

$$
1 / L=(1 / a+1 / b) / 2 .
$$

In other words, orthogonal dimensions are averaged using the harmonic mean rather than (for example) the geometric mean. Similar equations are easily found for other aperture shapes.

We now quantify more precisely our observation. that the diffractive beam spread will be linearly proportional to the product $(\lambda M R)$. For a uniformly illuminated circular aperture the diffraction pattern was first derived by Airy (Ref. 6, p. 396ff). In our notation, the half-angular size of the first dark ring in the Airy pattern, encircling 0.838 of the transmitted energy, is well known to be

$$
\theta=1.22 \lambda / D \text {. }
$$

Generalizing this, let $r$ now represent the radius of a circle containing 0.838 of the transmitted energy from a general aperture with beam quality $M$. Using Eq. (4) we obtain the relation

$$
0.838=1-\lambda M R(f / r) /\left(2 \pi^{2}\right) .
$$

Now, solve Eq. (16) for $r / f$, the half-angle containing almost $84 \%$ of the energy:

$$
r / f=0.31 \lambda M R \text {. }
$$

Using our definition of $L$ and letting $\theta$ now represent $(r / f)$, this can be written approximately as

$$
\theta=1.22 \lambda M / L \text {. }
$$

Equations (10) and (18) generalize Eq. (15) to arbitrary aperture shape and beam quality.

If the aperture is an unobscured circle and for $M$ equal to one, this reduces to Eq. (15). Also, for a square with side $a$ we obtain

$$
\theta=1.22 \lambda M / a \text {. }
$$

These results are consistent with our use of Eq. (10) for a characteristic aperture length and Eq. (18) for the angular beam spread from an aperture of arbitrary shape. Finally, note that it may be helpful in some cases to calculate the Fresnel number of an optical system using the characteristic length $L$ rather than the aperture diameter.

\section{Application of the Scaling Relation}

We have exercised the HELAWS code (containing BRLPRO and contained in EOSAEL) ${ }^{7}$ with the following questions in mind:

(1) How adequate is it to represent a centrally obscured and/or rectangular aperture by means of a circular aperture with the same overall area, power, and (therefore) intensity?

(2) Given that we would like to represent different aperture shapes and obscurations with our similarity transformation, how good a fix can be obtained in the presence of linear atmospheric effects such as turbulence and wind?

(3) How good is our transformation in the face of nonlinear effects, i.e., thermal blooming?

Figures 2-4 were generated with HELAWS using variations of the base case parameters listed in Table I. In each figure, curve 1 represents the results for a reference uniformly illuminated circular aperture of $1-\mathrm{m}$ diameter $D$, beam quality equal to one. Curve 2 presents the results for a uniformly illuminated circular aperture with a $10 \%$ central obscuration but with the same area and beam quality (i.e., $M=1$ ) as the base case. Since $F$ is 0.1 , Eq. (7) yields an aperture diameter $D^{\prime}$ of $1.054 \mathrm{~m}$. Finally, curve 3 presents the results for a uniformly illuminated circular aperture (no obscuration), equal area as before, but with beam quality (1.387) calculated from our scaling formula (or Fig. 1).

In other words, curve 2 corresponds to the real HEL aperture with $10 \%$ obscuration. Curve 1 is what we predict for an unobscured circular aperture matching only the area. Curve 3 results if we also match the scaling parameter $M^{*} R$. First, refer to Figs. 2 and 3, showing the $1 / e$ spot diameter and $1 / e$ peak fluence, respectively, in the absence of thermal blooming. Comparing curves 1 and 2 shows that simply trying to represent a $10 \%$ obscuration with a circular aperture of equal area would lead to gross errors. It is easy to show that there is a rectangular aperture $(L / W \sim 1.9)$ with the same area and value of $R$ as the $10 \%$ obscured circular aperture. Hence by the encircled energy theorem the rectangle will have the same asymptotic beam spread as the obscured circle (curve 2). Clearly, if one tries to represent such a rectangle with the unobscured circular aperture (curve 1), the same gross errors will result, even without thermal blooming.

However, for curve 3 (for which $M$ is set using the scaling relation) the match is excellent beyond $\sim 4300$ $\mathrm{m}$. This range defines the beginning of the asymptotic region for this example. Two things are worth noting here: (1) the $M^{*} R=$ const similarity relation works quite well becoming better as the range increases; (2) it works well even for propagation in the turbulent atmosphere.

Consulting Fig. 4 in which the thermally bloomed 


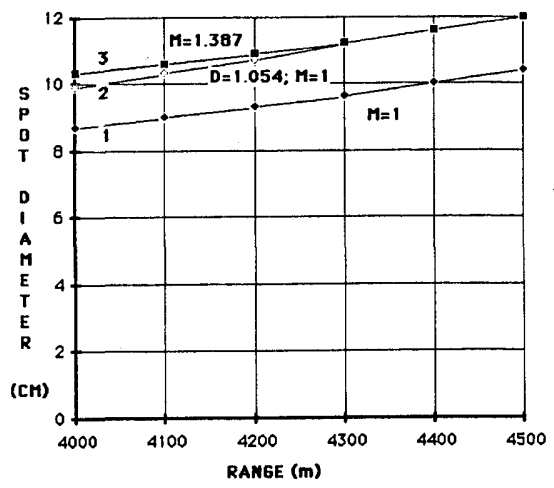

Fig. 2. Unbloomed spot diameter vs range.

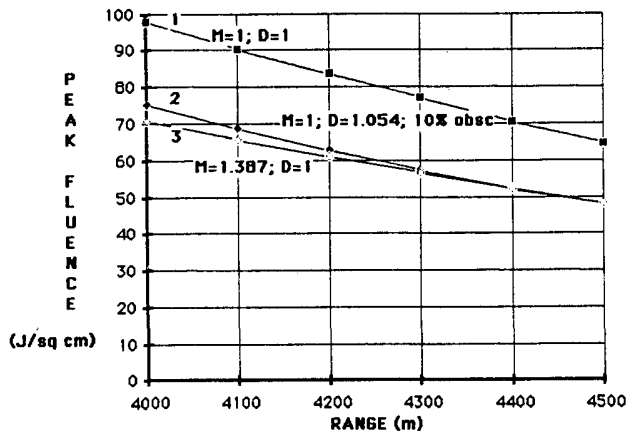

Fig. 3. Unbloomed peak fluence vs range.

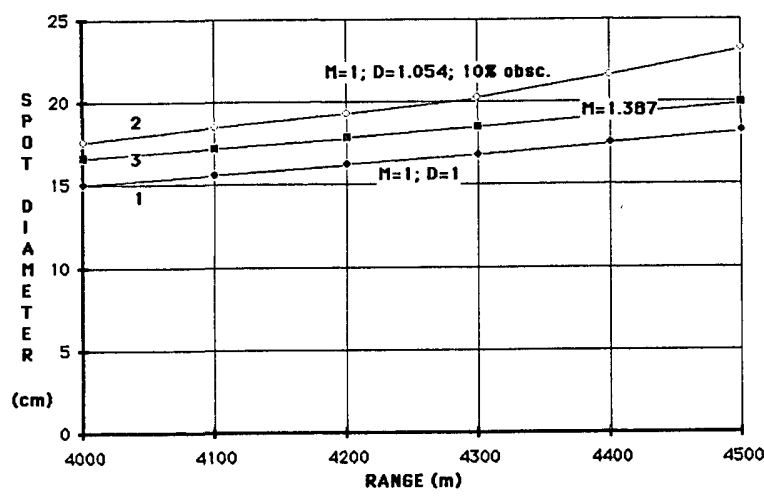

Fig. 4. Thermally bloomed $1 / e$ spot diameter vs range.

Table I. Input Quantities for HELAWS (BRLPRO) Code

\begin{tabular}{ll} 
Laser parameters & \\
Operating mode & Repetitively pulsed \\
Wavelength & $10.6 \mu \mathrm{m}$ \\
Total system jitter (1 sigma) & $10 \mu \mathrm{rad}$ \\
Variable focal range & Equal to target range \\
Energy per pulse & $10 \mathrm{~kJ}$ \\
Pulse repetition rate & $5 \mathrm{~Hz}$ \\
Pulse duration & $20 \mu \mathrm{s}$ \\
& \\
Atmospheric parameters & \\
Ambient temperature & $10^{\circ} \mathrm{C}$ \\
Relative humidity & $85 \%$ \\
Turbulence level & Moderate \\
Refractive-index structure const & $8.40 \times 10^{-14} \mathrm{~m}^{-2 / 3}$ \\
Magnitude of wind velocity & $1.0 \mathrm{~m} / \mathrm{s}$ \\
\hline
\end{tabular}

1/e spot diameter is plotted, we see that using the scaling relation (curve 3 ) gets us only about halfway to the $10 \%$ obscured aperture results (curve 2). However, the codes handle the $10 \%$ obscuration case already, and we are only looking for a means of extending the range of existing propagation codes to include larger obscurations and rectangular apertures.

\section{Conclusions}

The beam-quality/aperture-shape scaling relation has been derived under conditions most appropriate to free-space propagation for lasers with constant pupil function. Its extended usefulness has been demonstrated in an application to linear laser propagation within the turbulent atmosphere using a representative scaling law code (BRLPRO). In the presence of thermal blooming the scaling law remains partially effective and may still be useful as a partial fix. Our analysis has also provided a generalization of the Airy expression for the angular spread of light from a laser aperture of arbitrary shape and beam quality. New definitions for a characteristic aperture length and an aperture-quality factor allow comparison of diffraction characteristics of different aperture shapes, independent of wavelength.

The author would like to express his appreciation to P. Shugart, J. Morris, J. Fleck, and J. Reilly for their help in the study and to L. Ingber and E. Milne for comments on the manuscript. This work was supported by a contract from the U.S. Army TRADOC Operations Research Activity, White Sands Missile Range, NM.

\section{References}

1. F. G. Gebhardt and M. B. Richardson, "Nonlinear Aerosol Vaporization and Breakdown Effects Module-NOVAE," EOSAEL 84 22 (Draft Report), Atmospheric Sciences Laboratory, White Sands Missile Range, NM (Oct. 1984).

2. J. A. Fleck, Jr, J. R. Morris, and M. D. Feit, "Time-Dependent Propagation of High Energy Laser Beams through the Atmosphere," Appl. Phys. 10, 129 (1976); J. R. Morris, "Four-D Propagation Code for High-Energy Laser Beams: A User's Manual," UCID-17217, Lawrence Livermore National Laboratory (5 Aug. 1976).

3. H. J. Breaux, "Simplified Predictive Methodology for Nonlinear Repetitive Pulse and CW High Energy Laser Propagation," Proc. Soc. Photo-Opt. Instrum. Eng. 195, 192 (1979).

4. P. P. Clark, J. W. Howard, and E. R. Freniere, "Asymptotic Approximation to the Encircled Energy Function for Arbitrary Aperture Shapes," Appl. Opt. 23, 353 (1984).

5. A. Sommerfeld, Optics (Academic, New York, 1950), pp. 220-221.

6. M. Born and E. Wolf, Principles of Optics (Pergamon, New York, 1980).

7. P. Beavers, High Energy Laser Weapon Simulation (HELAWs), Analyst's Manual, Vol. 1, U.S. Army Materiel Systems Analysis Activity, Combat Support Division, Aberdeen Proving Grounds, MD (Sept. 1983). 